Series A

\author{
I. MATHEMATICA
}

382

\title{
ON SYSTEMS OF LINEAR AND QUADRATIC EQUATIONS IN FINITE FIELDS
}

BY

AIMO TIETÄVÄINEN 
Communicated 8 October 1965 by P. J. Myrberg and K. Inkeri. 


\section{On systems of linear and quadratic equations in finite fields}

1. Introduction. Let $K=G F(q)$ be a finite field of $q$ elements where $q=p^{n}, p$ is an odd prime and $n$ a positive integer. Consider the system

$$
\left\{\begin{array}{l}
\sum_{j=1}^{s} \alpha_{j} \xi_{j}^{2}=\alpha \\
\sum_{j=1}^{s} \beta_{i j} \xi_{j}=\beta_{i}(i=1, \ldots, t)
\end{array}\right.
$$

where $\alpha_{1}, \ldots, \alpha_{s}$ are non-zero, $\alpha, \beta_{1}, \ldots, \beta_{t}$ arbitrary elements of $K$, and the $\beta_{i j}$ 's are elements of $K$ such that the $t \times s$ matrix $\left(\beta_{i j}\right)$ has rank $t$. The purpose of this note is to prove the following result.

Theorem. The system (1) has a solution $\left(\xi_{1}, \ldots, \xi_{s}\right)$ in $K$ if $s=$ $2 t+2$. On the other hand, in case $s=2 t+1$ there exist, in every $K$, systems (1) which are insolvable in $K$.

This theorem has been proved by Dickson [4] in case $t=0$ and by Cohen ([2], remark 4; [3]) in case $t=1$. It is a conjecture of Cohen [2].

2. Preliminary remarks. Let $\sigma, \sigma_{1}, \ldots, \sigma_{v}$ be elements of $K$. Define the trace of $\sigma$ as

$$
\operatorname{tr}(\sigma)=\sigma+\sigma^{p}+\ldots+\sigma^{p-1}
$$

so that $\operatorname{tr}(\sigma)$ may be considered as an integer $(\bmod p)$. Define, furthermore,

$$
e(\sigma)=e^{2.7 i \operatorname{tr}(\sigma) / P} .
$$

Then we have

$$
e\left(\sum_{j=1}^{v} \sigma_{j}\right)=\prod_{j=1}^{v} e\left(\sigma_{j}\right)
$$

Consider the system

$$
f_{i}\left(\xi_{1}, \ldots, \xi_{s}\right)=\delta_{i}(i=1, \ldots, u)
$$


where the $f_{i}$ 's are polynomials with coefficients in $K$ and the $\delta_{i}$ 's are elements of $K$. It has been proved in [1] that the number of solutions $\left(\xi_{1}, \ldots, \xi_{s}\right)$ of the system (3) is equal to

$$
q^{-u} \sum_{\mathbf{c}} e\left(-\sum_{i=1}^{u} \gamma_{i} \delta_{i}\right) \sum_{\xi_{1}} \ldots \sum_{\xi_{s}} e\left(\sum_{i=1}^{u} \gamma_{i} f_{i}\left(\xi_{1}, \ldots, \xi_{s}\right)\right) .
$$

Here and hereafter, in the sums of type $\sum_{\mathbf{c}}$ the summation is over all the vectors $\mathbf{e}=\left(\gamma_{1}, \ldots, \gamma_{u}\right)$ with the $\gamma_{i}$ 's in $K$. Moreover, in the sums of type $\sum_{\xi}$ the variable runs through all the elements of $K$. By (2) and (4), the number of solutions of the system

$$
\sum_{j=1}^{s} f_{i j}\left(\xi_{j}\right)=\delta_{i} \quad(i=1, \ldots, u),
$$

where the $f_{i j}$ 's are polynomials over $K$, is equal to

$$
q^{-u} \sum_{\mathbf{c}} e\left(-\sum_{i=1}^{u} \gamma_{i} \delta_{i}\right) \prod_{j=1}^{s} \sum_{\xi_{j}} e\left(\sum_{i=1}^{u} \gamma_{i} f_{i j}\left(\xi_{j}\right)\right) .
$$

Let us denote

$$
S(\gamma, \delta)=\sum_{\xi} e\left(\gamma \xi^{2}+\delta \xi\right) .
$$

It is well known (see, for example, [2]) that $|S(\gamma, \delta)|=q^{1 / 2}$ if $\gamma \neq 0$.

3. Proof of the theorem. Let $s=2 t+2$. Then the number of solutions of the system (1) is, by (5), equal to

$$
N=q^{-t-1} \sum_{\mathbf{c}} e\left(-\varkappa \alpha-\sum_{i=1}^{t} \lambda_{i} \beta_{i}\right) \prod_{j=1}^{2 t+2} S\left(\varkappa \alpha_{j}, \sum_{i=1}^{t} \lambda_{i} \beta_{i j}\right)
$$

where $\mathbf{e}=\left(\varkappa, \lambda_{1}, \ldots, \lambda_{t}\right)$. We break up this summation into two parts according as $x=0$ or $\varkappa \neq 0$, writing

$$
N=q^{-t-1}\left(\sum_{x=0}+\sum_{x \neq 0}\right)=q^{-t-1}\left(U_{1}+l_{2}\right) .
$$

In case $t=0$ we have $U_{1}=q^{2}$. In case $t \geqq 1 L_{1}$ is, by (5), equal to $q^{t} N_{1}$ where $N_{1}$ is the number of solutions of the system

$$
\sum_{j=1}^{2 t+2} \beta_{i j} \xi_{j}=\beta_{i} \quad(i=1, \ldots, t) .
$$

Because the matrix $\left(\beta_{i j}\right)$ has rank $t$ then $N_{1}=q^{t+2}$. Consequently $U_{1}=q^{2 t+2}$, for every $t$. In the sum $U_{2}$ we have $x x_{j} \neq 0$, for every c. Therefore $\left|S\left(\varkappa \alpha_{j}, \sum_{i=1}^{t} \lambda_{i} \beta_{i j}\right)\right|=q^{1 / 2}$ and hence 


$$
\left|U_{2}\right| \leqq\left(q^{t+1}-q^{t}\right) q^{t+1}=q^{2 t+2}-q^{2 t+1}
$$

Consequently

$$
N \geqq q^{-t-1}\left(U_{1}-\left|U_{2}\right|\right) \geqq q^{t}>0 .
$$

This proves the former part of the theorem.

For the proof of the latter part of the theorem it is sufficient to note that the system

$$
\left\{\begin{array}{l}
-\sum_{j=1}^{t} \xi_{j}^{2}+\sum_{j=t+1}^{2 t+1} \xi_{j}^{2}=\alpha \\
\xi_{i}+\xi_{t+i}=0 \quad(i=1, \ldots, t),
\end{array}\right.
$$

where $\alpha$ is a non-square of $K$, is insolvable in $K$.

University of Turku

Turku, Finland

\section{References}

[1] CARlitz, L.: Invariant theory of systems of equations in a finite field. - J. Analyse Math. 3 (1954), 382-413.

[2] Cohen, E.: The number of simultaneous solutions of a quadratic equation and a pair of linear equations over a Galois field. - Rev. Math. Pures Appl. 8 (1963), 297-303.

[3] - - The number of planes contained in the complement of a quadric in an affine Galois space. - J. Tennessee Acad. Sci. 38 (1963), 133-134.

[4] Dickson, L. E.: Linear groups with an exposition of the Galois field theory. Dover (1958). 